$\overline{\text { Original }}$

\title{
Enhanced Antifungal Effect of the Selective Medium for the Detection of Legionella Species by a Combination of Cycloheximide, Amphotericin B and Thiabendazole
}

\author{
HIROAKI INOUE*, AKIKO NODA, TOMOKO TAKAMA, \\ TOMOKI ISHIMA AND KUNIO AGATA
}

Tsukuba Research Laboratories, Aquas Corporation, 4-4 Midorigahara, Tsukuba, Ibaraki 300-2646, Japan

Received 28 November 2005/Accepted 10 February 2006

\begin{abstract}
The accurate detection of Legionella from environmental water samples using conventional plate culture methods is often made difficult by the overgrowth of non-target microorganisms on the selective agar plates. Acid pretreatment is a very effective pretreatment to decrease the overgrowth of heterotrophic bacteria. However, acid pretreatment would not be expected to eliminate molds. We evaluated the effects of four kinds of antifungal agents, individually and in combination, on the growth of Legionella strains and molds. Consequently, it was demonstrated that the combination of cycloheximide, amphotericin $B$ and thiabendazole was very effective in eliminating molds on agar plates, and resulted in the improved detection of Legionella. Examination of 214 cooling tower water samples using the enhanced antifungal selective medium (CAT $\alpha$ ) instead of GVPC $\alpha$ selective medium demonstrated a decrease in contamination by molds from $13.6 \%$ to $1.9 \%$ without affecting the growth of Legionella.
\end{abstract}

Key words : Antifungal agent/Detection/Legionella/Selective medium/Thiabendazole.

\section{INTRODUCTION}

Legionella species are gram-negative bacteria ubiquitously found in natural and man-made water systems. Human inhalation of aerosolized water from Legionella contaminated sources, such as cooling tower water or bath water, can result in a severe form of pneumonia called Legionnaires' disease (Vogel and Isberg, 1999). Therefore, the control and management of Legionella contamination in water systems is very important, and the effectiveness of these efforts must be monitored by screening for the presence of Legionella. There are several methods to detect Legionella from environmental water samples including plate culture, fluorescent antibody staining, and gene detection. Of these, the plate culture method is the standard method (ISO11731, 1998) for the detection of Legionella.

${ }^{*}$ Corresponding author. Tel : +81-29-847-6000, Fax: + 8129-847-6080
However, the detection of Legionella in environmental water samples using conventional plate culture is often inhibited by the overgrowth of non-target microorganisms on the agar plates. Therefore, pretreatment of the samples and the use of a selective medium are necessary. The samples are treated with acid buffer (Bopp et al., 1981; ISO11731, 1998), heat (Dennis et al., 1984; ISO11731, 1998) or a combination of heat and acid treatment (Kasuga et al., 1999), and the treated samples are inoculated onto agar plates containing antibiotics as selective agents. Acid treatment is a very effective pretreatment to eliminate non-target heterotrophic bacteria. We recently reported that the use of the acid-phosphate buffer instead of the $\mathrm{HCl}-\mathrm{KCl}$ buffer further decreased the overgrowth of non-target heterotrophic bacteria (Inoue et al., 2004). However, an acid pretreatment would not be expected to eliminate molds because some molds are resistant to low $\mathrm{pH}$, and therefore, the use of a selective medium containing antifungal agents may be effective in eliminating molds.

The addition of antifungal antibiotics such as 
amphotericin B (Greaves, 1980; Okuda et al., 1984), anisomycin (Edelstein, 1981; 1982), cycloheximide (Bopp et al., 1981; Dennis et al., 1984) and natamycin (Edelstein et al., 1996) has been reported as a method for the selection of Legionella. However, the detection of Legionella in environmental water samples is sometimes inhibited by the overgrowth of molds even when using a selective medium. From our previous results using GVPC $\alpha$ selective agar plates, $654(7.8 \%)$ of 8400 water samples (collected mainly from cooling towers, whirlpool baths and spas between September 2003 and August 2004) were contaminated by molds.

In this paper, we report the effect of thiabendazole as a selective antifungal agent, and we describe an improved selective medium to eliminate the overgrowth of molds on agar plates for the detection of Legionella by including a combination of cycloheximide, amphotericin $\mathrm{B}$ and thiabendazole.

\section{MATERIALS AND METHODS}

\section{Legionella and mold strains, and growth condi- tions}

The Legionella strains used were the following: Legionella pneumophila Philadelphia-1 ATCC33152; L. bozemanii ATCC33217; L. longbeachae ATCC33462; L. micdadei ATCC33218; L. gormanii ATCC33297; and L. anisa ATCC35292. These strains were cultured on a buffered charcoal yeast extract supplemented with $\alpha$-ketoglutarate (BCYE $\alpha$ ) agar (Edelstein, 1981) at $37^{\circ} \mathrm{C}$. Ten strains of molds, which had been isolated from cooling tower water samples, were grown on a potato dextrose agar (PDA) plates (4 strains) and GVPC $\alpha$ selective agar plates (6 cycloheximide resistant strains. These strains which had previously inhibited our examination of Legionella detection), were used to evaluate the antifungal effect of each type of agar medium. The molds were precultured on PDA plates at $27{ }^{\circ} \mathrm{C}$ for 2 weeks. These mold strains were identified by microscopic observation (Malloch, 1981).

\section{Antifungal agents}

Cycloheximide (Wako Pure Chemical Industries), amphotericin B (Sigma), griseofulvin (Wako Pure Chemical Industries) and thiabendazole (Sigma) were used as antifungal agents. These antifungal agents were dissolved and diluted in dimethyl sulfoxide (DMSO) to appropriate concentrations.

\section{Evaluation of the antimicrobial activity against Legionella strains}

Legionella strains were precultured overnight in buffered yeast extract broth (BYEB) (Liebers et al., 1989) at $37^{\circ} \mathrm{C}$, and $500 \mu \mathrm{l}$ of each broth was added to $50 \mathrm{ml}$ of fresh BYEB (approximately $10^{6} \mathrm{cells} / \mathrm{ml}$ ). The solutions $(5 \mu \mathrm{l})$ of antifungal agents were mixed with the broths of Legionella strains (995 $\mu$ l each) in 48-well micro plates. After 3 days of incubation at 37 ${ }^{\circ} \mathrm{C}$, the growth of Legionella was judged from the turbidity of the broth.

\section{Selective agar plates}

We prepared six types of agar plates all based on GVP $\alpha$ agar (BCYE $\alpha$ containing $3 \mathrm{~g} /$ liter of glycine, $5 \mathrm{mg} /$ liter of vancomycin, 100000 units/liter of polymyxin B). GVPC $\alpha$ (GVP $\alpha$ containing $80 \mathrm{mg} / \mathrm{li}-$ ter of cycloheximide), GVPA $\alpha$ (GVP $\alpha$ containing 80 $\mathrm{mg} /$ liter of amphotericin B), GVPG $\alpha$ (GVP $\alpha$ containing $80 \mathrm{mg} /$ liter of griseofulvin) and GVPT $\alpha$ (GVP $\alpha$ containing $40 \mathrm{mg} /$ liter of thiabendazole) agar plates were tested for the ability to inhibit the growth of molds. Also, CAT $\alpha$ (GVP $\alpha$ containing 80 $\mathrm{mg} /$ liter of cycloheximide, $80 \mathrm{mg} /$ liter of amphotericin $B$ and $40 \mathrm{mg} /$ liter of thiabendazole) agar plates were prepared as a selective medium with enhanced antifungal activity.

\section{Evaluation of each selective medium}

The spores of 10 strains of molds were suspended to appropriate concentrations in $10 \mathrm{ml}$ of sterile deionized water by using a nichrome wire loop. One hundred microliters of each suspension was inoculated onto five types of agar plates (GVP $\alpha$, GVPC $\alpha$, GVPA $\alpha$, GVPG $\alpha$ and GVPT $\alpha$ ). After 8 days of incubation at $37{ }^{\circ} \mathrm{C}$, the growth of molds on each agar medium was recorded.

Next, L. pneumophila cells were suspended in sterile deionized water to a concentration of approximately $10^{2} \mathrm{CFU} / \mathrm{ml}$; each mold spore suspension and L. pneumophila suspension were mixed. Two hundred microliters of each mixture was inoculated on four types of agar plates (GVPC $\alpha$, GVPA $\alpha$, GVPT $\alpha$ and CAT $\alpha$ ). After 8 days of incubation at $37^{\circ} \mathrm{C}$, the growth of the molds and L. pneumophila on each agar medium was recorded.

Next, we compared and evaluated the growth of Legionella colonies on BCYE $\alpha$, GVPC $\alpha$ and CAT $\alpha$ agar plates. Legionella strains were suspended in sterile deionized water to a concentration of approximately $10^{3} \mathrm{CFU} / \mathrm{ml}$. One hundred microliters of each cell suspension was inoculated on each of three plates (BCYE $\alpha$, GVPC $\alpha$ and CAT $\alpha$ ). After 8 days of incubation at $37^{\circ} \mathrm{C}$, the colonies were enumerated. 
TABLE 1. Antimicrobial activity of antifungal agents against Legione/la species.

\begin{tabular}{lccccc}
\hline \multirow{2}{*}{ Strain } & ATCC No. & \multicolumn{4}{c}{ MIC value $(\mu \mathrm{g} / \mathrm{ml})$} \\
\cline { 2 - 5 } & & Cycloheximide & Amphotericin B & Griseofulvin & Thiabendazole \\
\hline L. pneumophila & 33152 & $>200$ & $>200$ & $>200$ & 100 \\
L. bozemanii & 33217 & $>200$ & $>200$ & $>200$ & 100 \\
L. longbeachae & 33462 & $>200$ & $>200$ & $>200$ & 100 \\
L. micdadei & 33218 & $>200$ & $>200$ & $>200$ & 200 \\
L. gormanii & 33297 & $>200$ & $>200$ & $>200$ & 200 \\
L. anisa & 35292 & $>200$ & $>200$ & $>200$ & 100 \\
\hline
\end{tabular}

\section{Enumeration of Legionella in environmental wa- ter samples}

Legionella were enumerated according to the standard method (ISO11731, 1998). Collected water samples (214 samples of cooling tower water) were concentrated 100 -fold by centrifugation. The concentrated samples were pretreated with acid buffer. In this case, $500 \mu \mathrm{l}$ of the acid-phosphate buffer (Inoue et al., 2004) was added to $500 \mu \mathrm{l}$ of the concentrated sample in a sterile test tube with a cap at room temperature $\left(25^{\circ} \mathrm{C}\right)$. After $10 \mathrm{~min}, 200 \mu \mathrm{l}$ portions were inoculated onto GVPC $\alpha$ and CAT $\alpha$ selective agar plates. The plates were incubated at $37{ }^{\circ} \mathrm{C}$ for 8 days, and Legionella colonies on both types of agar plates were enumerated.

\section{RESULTS}

\section{Effect of antifungal agents on Legionella strains}

We evaluated the effect of cycloheximide, amphotericin B, griseofulvin and thiabendazole on Legionella strains (Table 1). Thiabendazole showed weak activity against Legionella with a minimum inhibitory concentration (MIC) of 100 to $200 \mu \mathrm{g} / \mathrm{ml}$, but had no effect on the growth of Legionella at concentrations below $100 \mu \mathrm{g} / \mathrm{ml}$. In contrast, cycloheximide, amphotericin B and griseofulvin had no effect on Legionella.

\section{Effect of antifungal agents against molds}

Next, we tested the effect of the antifungal agents in five types of selective agar plates on the growth of molds (Table 2). Both GVP $\alpha$ (without antifungal agents) and GVPG $\alpha$ (with griseofulvin) plates were overgrown with molds. GVPC $\alpha$ (with cycloheximide) was able to inhibit the growth of only 2 strains of molds, since 6 of the mold strains tested were resistant to cycloheximide. In contrast, GVPA $\alpha$ (with amphotericin B) and GVPT $\alpha$ (with thiabendazole) were able to inhibit the growth of 5 and 3 strains of molds, respectively. The results in Table 2 demonstrate that amphotericin B and thiabendazole each had a different antifungal spectrum.

\section{Effect of CAT $\alpha$ selective medium on the detec- tion of L. pneumophila}

Based on the results of the foregoing experiments, we expected to increase the antifungal effect of the selective medium by the combination of cycloheximide, amphotericin $B$ and thiabendazole. We therefore prepared CAT $\alpha$ selective medium containing these antifungal agents. Table 3 shows the detection of $L$. pneumophila on 4 types of selective media. CAT $\alpha$ selective medium allowed the detection of $L$. pneumophila colonies without the contamination of molds. In contrast, GVPC $\alpha$, GVPA $\alpha$ and GVPT $\alpha$ selective media were severely contaminated by the growth of 7,2 and 6 strains of molds, respectively.

TABLE 2. Inhibition of mold growth on each selective medium.

\begin{tabular}{|c|c|c|c|c|c|c|}
\hline \multirow{2}{*}{ No. } & \multirow{2}{*}{ Strain } & \multicolumn{5}{|c|}{ Growth of molds on each agar plate ${ }^{a}$} \\
\hline & & $\operatorname{GVP} \alpha$ & GVPC $\alpha$ & GVPA $\alpha$ & GVPG $\alpha$ & GVPT $\alpha$ \\
\hline M1 & Penicillium sp. & ++ & ++ & - & ++ & ++ \\
\hline M2 & Trichoderma sp. & ++ & - & + & ++ & - \\
\hline M3 & Aspergillus sp. & ++ & - & - & ++ & ++ \\
\hline M4 & Penicillium sp. & ++ & ++ & + & ++ & - \\
\hline M5 & Fusarium sp. & ++ & ++ & ++ & ++ & + \\
\hline M6 & Aspergillus sp. & ++ & ++ & + & ++ & ++ \\
\hline M7 & Aspergillus sp. & ++ & ++ & - & ++ & ++ \\
\hline M8 & Aspergillus sp. & ++ & ++ & - & ++ & ++ \\
\hline M9 & Penicillium sp. & ++ & ++ & - & ++ & ++ \\
\hline M10 & Penicillium sp. & ++ & ++ & ++ & ++ & - \\
\hline
\end{tabular}

${ }^{a}++$, Overgrowth; + , growth inhibition; -, no growth 
TABLE 3. Detection of $L$. pneumophila on each selective medium.

\begin{tabular}{|c|c|c|c|c|c|}
\hline \multirow{2}{*}{ No. } & \multirow{2}{*}{ Strain } & \multicolumn{4}{|c|}{ Colony counts of $L$. pneumophila on each agar plate (CFU/plate) } \\
\hline & & GVPC $\alpha$ & GVPA $\alpha$ & GVPT $\alpha$ & CAT $\alpha$ \\
\hline M1 & Penicillium sp. & 23 & 42 & $O G$ & 52 \\
\hline M2 & Trichoderma sp. & 42 & 26 & 39 & 35 \\
\hline M3 & Aspergillus sp. & 19 & 26 & $O G$ & 41 \\
\hline M4 & Penicillium sp. & $O G^{a}$ & 15 & 18 & 16 \\
\hline M5 & Fusarium sp. & 6 & $O G$ & 12 & 44 \\
\hline M6 & Aspergillus sp. & 4 & 17 & $O G$ & 39 \\
\hline M7 & Aspergillus sp. & OG & 22 & OG & 22 \\
\hline M8 & Aspergillus sp. & $O G$ & 20 & OG & 31 \\
\hline M9 & Penicillium sp. & $O G$ & 32 & $O G$ & 35 \\
\hline M10 & Penicillium sp. & OG & OG & 35 & 30 \\
\hline
\end{tabular}

${ }^{a}$ Overgrowth of the molds

The growth inhibition of each prepared agar medium against Fusarium species (M5) is shown in Figure 1. Thus, the synergistic effect of a combination of cycloheximide, amphotericin B and thiabendazole against molds was demonstrated.

Next, we examined the growth of Legionella colonies on CAT $\alpha$ selective medium. Table 4 shows a comparison of the colony counts of Legionella on $\operatorname{BCYE} \alpha$, GVPC $\alpha$, and CAT $\alpha$ selective media. No difference in the percentage of colony forming units
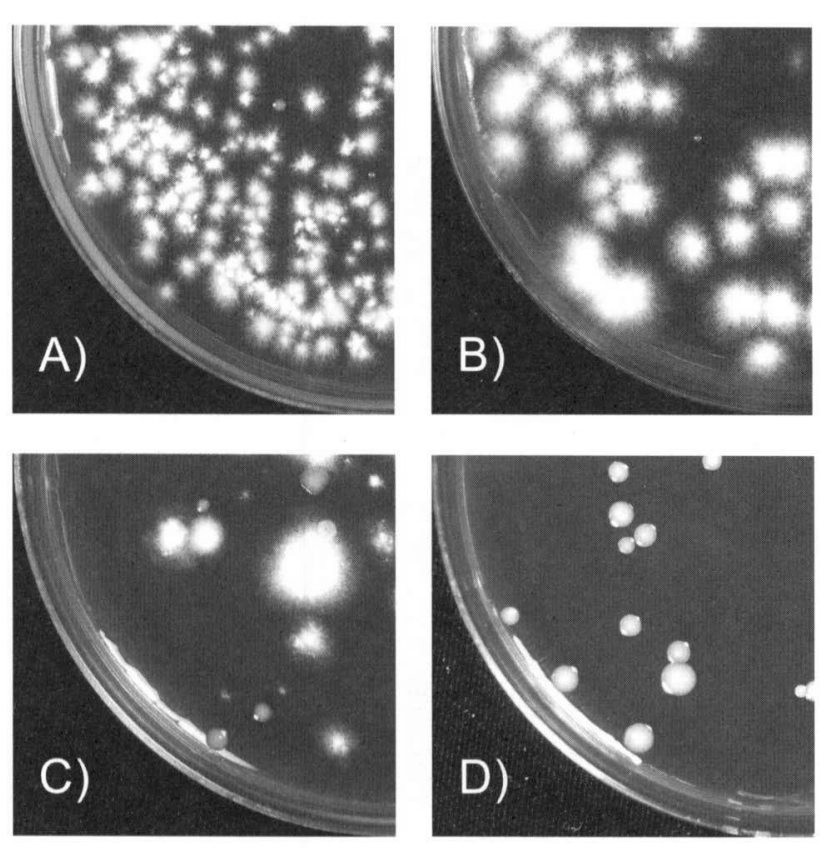

FIG. 1. Growth inhibition of each prepared agar plate against Fusarium species (M5). The photographs show the growth of Fusarium species and L. pneumophila after 8 days of incubation at $37^{\circ} \mathrm{C}$. A), GVPC $\alpha$, containing 80 $\mu \mathrm{g} / \mathrm{ml}$ of cycloheximide; B), GVPA $\alpha$, containing $80 \mu \mathrm{g} /$ $\mathrm{ml}$ of amphotericin B; C), GVPT $\alpha$, containing $40 \mu \mathrm{g} / \mathrm{ml}$ of thiabendazole; D), CAT $\alpha$, containing $80 \mu \mathrm{g} / \mathrm{ml}$ of cycloheximide, $80 \mu \mathrm{g} / \mathrm{ml}$ of amphotericin B, and $40 \mu \mathrm{g} /$ $\mathrm{ml}$ of thiabendazole. on GVPC $\alpha$ and CAT $\alpha$ selective media was observed.

\section{Detection of Legionella using CAT $\alpha$ selective medium}

From the results shown in Tables 3 and 4, the CAT $\alpha$ selective medium is very effective in inhibiting the growth of molds and supporting the growth of Legionella. Accordingly, we evaluated the validity of CAT $\alpha$ selective medium by comparing it with GVPC

TABLE 4. Comparison of the growth of Legionella on BCYE $\alpha$, GVPC $\alpha$ and CAT $\alpha$ selective media

\begin{tabular}{lcccc}
\hline \multirow{2}{*}{\multicolumn{1}{c}{ Strain }} & ATCC No. & \multicolumn{3}{c}{$\begin{array}{c}\text { Recovery of Legionella } \\
\text { colonies on: }\end{array}$} \\
\cline { 2 - 5 } & & BCYE $\alpha$ & GVPC $\alpha$ & CAT $\alpha$ \\
\hline L. pneumophila & 33152 & 100 & 74 & 78 \\
L. bozemanii & 33217 & 100 & 10 & 14 \\
L. longbeachae & 33462 & 100 & 85 & 83 \\
L. micdadei & 33218 & 100 & 71 & 83 \\
L. gormanii & 33297 & 100 & 4 & 3 \\
L. anisa & 35292 & 100 & 40 & 44
\end{tabular}

${ }^{a}$ The value shows the percentage of colony forming units of Legionella (two replicate plates) when the colony counts on BCYE $\alpha$ were $100 \%$. Initial cell counts of strains in each cell suspension were the following: L. pneumophila, $2 \times \quad 10^{3} \mathrm{CFU} / \mathrm{ml} ;$ L. bozemanii, $1 \times 10^{3} \mathrm{CFU} / \mathrm{ml} ; L$. longbeachae, $3 \times 10^{3} \mathrm{CFU} / \mathrm{ml} ; \quad$ L. micdadei, $6 \times 10^{3}$ $\mathrm{CFU} / \mathrm{ml}$; L. gormanii, $4 \times 10^{3} \mathrm{CFU} / \mathrm{ml}$; L. anisa, $2 \times 10^{2}$ $\mathrm{CFU} / \mathrm{ml}$.

TABLE 5. Comparison of mold contamination on GVPC $\alpha$ and CAT $\alpha$ selective media

\begin{tabular}{lrr}
\hline \multicolumn{1}{c}{ Result } & GVPC $\alpha$ & CAT $\alpha$ \\
\hline Growth of molds & $21(9.8 \%)$ & $3(1.4 \%)$ \\
Overgrowth of molds $^{a}$ & $8(3.7 \%)$ & $1(0.5 \%)$ \\
\hline Total & $29(13.6 \%)$ & $4(1.9 \%)$ \\
\hline
\end{tabular}

$n=214$

a The growth of molds over more than a third of the agar plate 


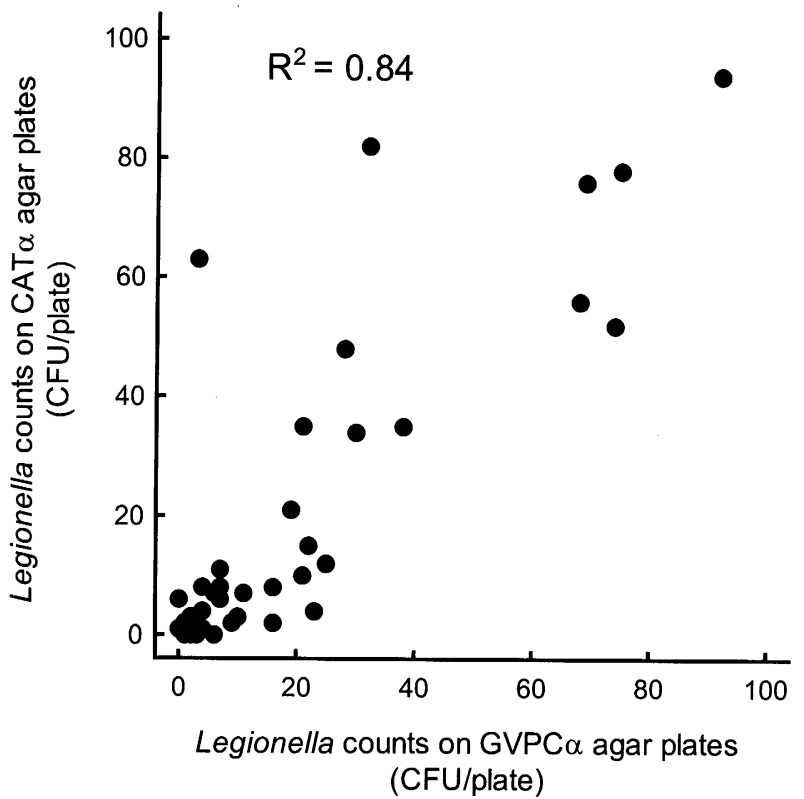

FIG. 2. Comparison of Legionella counts detected on GVPC $\alpha$ and CAT $\alpha$ selective media $(n=52)$

$\alpha$ selective medium. Table 5 shows the comparison of mold contamination on GVPC $\alpha$ and CAT $\alpha$ selective media. Out of a total of 214 cooling tower water samples, 29 samples (13.6 \%) were contaminated with molds on GVPC $\alpha$ selective medium. In contrast, four $(1.9 \%)$ of these 214 samples were contaminated with molds on CAT $\alpha$ selective medium. Furthermore, of these samples, eight (3.7\%) were overgrown with molds which covered more than a third of the GVPC $\alpha$ agar plate. In contrast, only one sample $(0.5 \%)$ was overgrown with molds on CAT $\alpha$ selective medium. Figure 2 shows the number of Legionel/a colonies on the GVPC $\alpha$ and CAT $\alpha$ agar plates plotted as a scatter diagram. The samples with no colonies on both types of selective media were eliminated after this figure was plotted. From these results, a high coefficient of correlation $\left(R^{2}=0.84\right)$ was seen.

\section{DISCUSSION}

The conventional plate culture method is a very important technique for the detection of Legionella because it is the currently used standard method. However, we need to improve the accuracy of the plate culture method.

Many colonies of molds inhibit the growth of Legionella on agar plates. Certain types of molds produce antibiotics that can inhibit the growth of Legionella on agar plates, and as a result, Legionella numbers may be underestimated. Therefore, antifungal agents were added to the selective agar plates to improve the accuracy of Legionella detection.

Lin et al. (1999) described the addition of fluconazole to standard Legionella-selective medium for eliminating yeasts, and Kasuga et al. (2002) reported the addition of amphotericin B to MWY selective medium for eliminating molds. Here, we report for the first time that thiabendazole was useful as an antifungal agent in the selective medium for the detection of Legionella, and show the validity of combining cycloheximide, amphotericin $B$ and thiabendazole.

In another experiment, we tested higher concentrations of cycloheximide in GVPC $\alpha$ agar plates for the inhibition of mold growth. However, $200 \mu \mathrm{g} / \mathrm{ml}$ of cycloheximide did not inhibit the growth of molds (data not shown). Therefore, a combination of antifungal agents was tested for the inhibition of the growth of molds.

Cycloheximide and amphotericin B are known to have no activity against bacteria. This is because cycloheximide is a glutarimide antibiotic that selectively inhibits eucaryotic protein synthesis, and amphotericin B is a polyene macrolide antibiotic that binds ergosterol in the cell membrane and results in the release of potassium and other ions, disrupting the function of the cell membrane. Allen and Gottlieb (1970) reported that thiabendazole inhibits the terminal electron transport system of mitochondria. Therefore, these antifungal agents have high selective toxicity against molds.

The results in Table 4 show that the percentages of Legionella colony forming units on GVPC $\alpha$ and CAT $\alpha$ selective media were lower than that on BCYE $\alpha$ medium. These data agrees with the report of Okuda et al. (1984). Based on these results, it is assumed that the effects were not due to the antifungal agents but to the antibiotics vancomycin and polymyxin $\mathrm{B}$.

We added $40 \mu \mathrm{g} / \mathrm{ml}$ of thiabendazole into the medium, an addition that had no effect on the growth of Legionella. Higher concentrations of thiabendazole may have no effect on the growth of Legionella, but our results indicate that $40 \mu \mathrm{g} / \mathrm{ml}$ is sufficient to inhibit the growth of molds (Tables 3 and 5).

In this study, we demonstrated that the combination of three antifungal agents with different mechanisms of action is very effective for inhibiting the growth of molds on the selective medium. We believe that use the CAT $\alpha$ selective medium improves the accuracy of Legionella detection from environmental water samples due to the reduced contamination with molds. 


\section{ACKNOWLEDGEMENTS}

We thank Tomoyuki Iwasawa and Yuuji Saruwatari for their support and helpful discussions.

\section{REFERENCES}

Allen, P. M., and Gottlieb, D. (1970) Mechanism of action of the fungicide thiabendazole, 2-(4'-tiazolyl) benzimidazole. Appl. Microbiol., 20, 919-926.

Bopp, C. A., Sumner, J. W., Morris, G. K., and Wells, J. G. (1981) Isolation of Legionella spp. from environmental water samples by low-pH treatment and use of a selective medium. J. Clin. Microbiol., 13, 714-719.

Dennis, P. J., Bartlett, C. L. R., and Wright, A. E. (1984) Comparison of isolation methods for Legionella spp.. Proceedings of the 2nd international symposium, Washington DC, Am. Soc. Microbiol., 294-296.

Dennis, P. J., Green, D., and Jones, B. P. C. (1984) A note on the temperature tolerance of Legionella. J. Appl. Bacteriol., 56, 349-350.

Edelstein, P. H. (1981) Improved semiselective medium for isolation of Legionella pneumophila from contaminated clinical and environmental specimens. J. Clin. Microbiol., 14, 298-303.

Edelstein, P. H. (1982) Comparative study of selective media for isolation of Legionella pneumophila from portable water. J. Clin. Microbiol., 16, 697-699.

Edelstein, P. H., and Edelstein, M. A. C. (1996) Natamycin as a selective antifungal agent in media for growth of Legionella spp.. J. Clin. Microbiol., 34, 185-187.

Greaves, P. H. (1980) New methods for the isolation of
Legionella pneumophila. J. Clin. Pathol., 33, 581-584.

International Organization for Standardization (ISO). (1998) Water quality - Detection and enumeration of Legionella. ISO11731.

Inoue, H., Iwasawa, T., Saruwatari, Y., and Agata, K. (2004) Improved acid pretreatment for the detection of Legionella species from environmental water samples using the plate culture method. Biocontrol Sci., 9, 43-50.

Kasuga, O., Takagi, K., Tani, K., and Kinumaki, A. (1999) Studies on detection methods for Legionella species from environmental water (in Japanese). Kansenshogaku Zasshi, 73, 25-34.

Kasuga, O., Misawa, H., Takagi, K., Tani, K., and Ikedo, M. (2002) Studies on efficient methods for detection of Legionella species from environmental water by use of its selective media (in Japanese). Kansenshogaku Zasshi, 76, 41-50.

Liebers, D. M., Baltch, A. L., Smith, R. P., Hammer, M. C., and Conroy, J. V. (1989) Susceptibility of Legionella pneumophila to eight antimicrobial agents including four macrolides under different assay condition. J. Antimicrob. Chemother., 23, 37-41.

Malloch, David. (1981) Moulds: their isolation, cultivation, and identification. University of Toronto press, Canada.

Okuda, K., Ikedo, M., and Yabuuti, E. (1984) WadowskyYee-Okuda (WYO) medium: a new selective medium for the isolation of legionellae strains from environmental water specimens (in Japanese). Kansenshogaku Zasshi, 58, 1073-1082.

Vogel, J. P., and Isberg, R. R. (1999) Cell biology of Legionella pneumophila. Curr. Opin. Microbiol., 2, 30-34. 